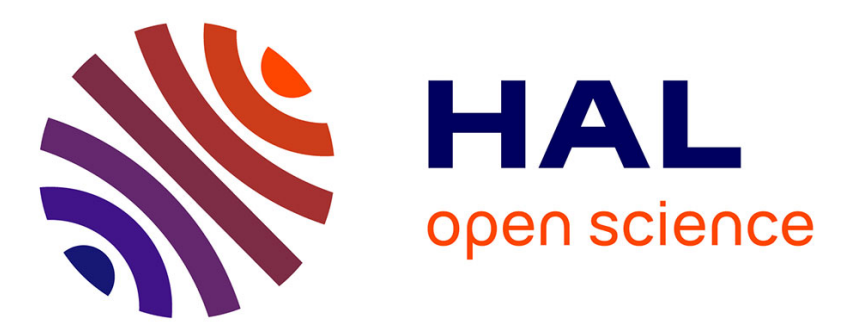

\title{
Improved Complexity for Power Edge Set Problem
}

\author{
Benoit Darties, Annie Chateau, Rodolphe Giroudeau, Mathias Weller
}

\section{To cite this version:}

Benoit Darties, Annie Chateau, Rodolphe Giroudeau, Mathias Weller. Improved Complexity for Power Edge Set Problem. IWOCA: International Workshop on Combinatorial Algorithms, Jul 2017, Newcastle, Australia. pp.128-141, 10.1007/978-3-319-78825-8_11 . hal-01715909

\section{HAL Id: hal-01715909 https://hal.science/hal-01715909}

Submitted on 23 Feb 2018

HAL is a multi-disciplinary open access archive for the deposit and dissemination of scientific research documents, whether they are published or not. The documents may come from teaching and research institutions in France or abroad, or from public or private research centers.
L'archive ouverte pluridisciplinaire HAL, est destinée au dépôt et à la diffusion de documents scientifiques de niveau recherche, publiés ou non, émanant des établissements d'enseignement et de recherche français ou étrangers, des laboratoires publics ou privés. 


\title{
Improved Complexity for Power Edge Set Problem
}

\author{
B. Darties ${ }^{1}$ and A. Chateau ${ }^{2}$ and R. Giroudeau ${ }^{2}$ and M. Weller ${ }^{2}$ \\ 1 Le2i FRE2005, CNRS, Arts et Métiers, Univ. Bourgogne Franche-Comté \\ 2 LIRMM - CNRS UMR 5506 - Montpellier, France \\ \{chateau, giroudeau, weller\}@lirmm.fr, \{benoit.darties\}@u-bourgogne.fr
}

\begin{abstract}
We study the complexity of Power Edge Set (PES), a problem dedicated to the monitoring of an electric network. In such context we propose some new complexity results. We show that PES remains $\mathcal{N P}$-hard in planar graphs with degree at most five. This result is extended to bipartite planar graphs with degree at most six. We also show that PES is hard to approximate within a factor lower than 328/325 in the bipartite case (resp. $17 / 15-\epsilon$ ), unless $\mathcal{P}=\mathcal{N} \mathcal{P}$, (resp. under $\mathcal{U G C}$ ). We also show that, assuming $\mathcal{E} \mathcal{T} \mathcal{H}$, there is no $2^{o(\sqrt{n})}$-time algorithm and no $2^{o(k)} n^{O(1)}$-time parameterized algorithm, where $n$ is the number of vertices and $k$ the number of PMUs placed. These results improve the current best known bounds.
\end{abstract}

\section{Introduction}

Monitoring the nodes of an electrical network can be carried out by means of Phasor Measurement Units (PMUs). The problem of placing an optimal number of PMUs on the nodes for complete network monitoring, is known as PowER Dominating Set [16]. A recent variant of the problem [15], called Power Edge SET (PES), is to have the PMUs on the network links rather than the nodes, considering the following two rules: (1) two endpoints of an edge bearing a PMU are monitored and (2) if one node is monitored and all but one of its neighbors are too, then the unmonitored neighbor becomes monitored. The problem of assigning a minimum number of PMUs to monitor the whole network is known to be $\mathcal{N} \mathcal{P}$-hard in the general case but can be solved in linear time on trees [15]. In this paper, we present some new complexity results, proposing new lower bounds according to classic complexity hypotheses.

We model the electrical network by a graph $G=(V, E)$ with $|V|=n$ and $|E|=m$. We let $V(G)$ and $E(G)$ denote the respective sets of vertices and edges of $G$. Further, $N_{G}(v)$ denotes the set of neighbors of $v$ and $d_{G}(v)=\left|N_{G}(v)\right|$ its degree in $G$. Finally, we let $N_{G}[v]:=N_{G}(v) \cup\{v\}$ denote the closed neighborhood of $v$ in $G$.

The problem Power EDGE SET can be seen as a problem of color propagation with colors 0 (white) and 1 (black), respectively designating the states not monitored and monitored of a vertex of $G$. Let $G=(V, E)$ be a graph as the 


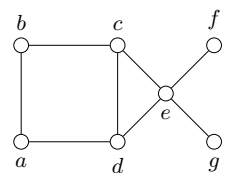

(a)

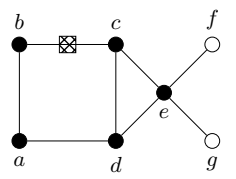

(e)

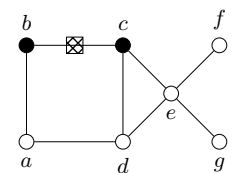

(b)

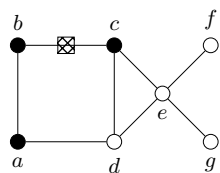

(c)

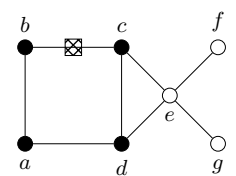

(d)

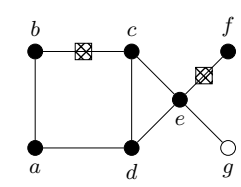

(f)

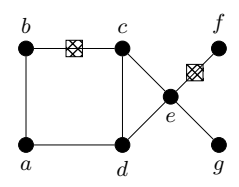

(g)

Fig. 1. Before placing any PMU (represented by crossed boxes on edges), all vertices are white (Fig. 1a). If we place a PMU on $\{b, c\}$, then $c(b)=c(c)=1$ (black) by Rule $R_{1}$ (Fig. 1b). By applying Rule $R_{2}$ on $b$, we obtain $c(a)=1$ (Fig. 1c). Then, Rule $R_{2}$ on $a$ gives $c(d)=1$ (Fig. 1d), and, finally, $c(e)=1$ with Rule $R_{2}$ on $c$ or $d$ (Fig. 1e). The color propagation is stopped, and we need to place a second PMU. A PMU on $\{e, f\}$ implies $c(f)=1$ by RULE $R_{1}$ (Fig. 1f) and Rule $R_{2}$ on $e$ gives $c(g)=1$ (Fig. 1g).

input of Power Edge Set and, for each vertex $v \in V$, let $c(v)$ be the color assigned to $v$ (we abbreviate $\bigcup_{v \in X} c(v)=: c(X)$ ). Before placing the PMUs, we have $c(V)=\{0\}$. Given a set $E^{\prime} \subseteq E$ of edges on which to place PMUs, colors propagate according to the following rules:

RuLE $R_{1}:$ if $(u, v) \in E^{\prime}$, then $c(u)=c(v)=1$ ("the endpoints of all $\{u, v\} \in E^{\prime}$ are colored").

Rule $R_{2}$ : for $u, u^{\prime}$ with $c(u)=1, u^{\prime} \in N_{G}(u)$ and $c(v)=1$ for all $v \in$ $N_{G}(u) \backslash\left\{u^{\prime}\right\}$, then $c\left(u^{\prime}\right)=1$ ("if $u^{\prime}$ is the only uncoloured neighbor of an already colored vertex $u$, then $u^{\prime}$ is colored" - we say that we apply RULE $R_{2}$ on $u$ to color $u^{\prime}$, or that $u^{\prime}$ is colored by propagation of $u$ ).

The objective of Power EDge SET is to find a smallest set of edges $E^{\prime} \subseteq E$ on which to place the PMUs such that $\mathrm{c}(\mathrm{V})=\{1\}$ after exhaustive application of Rule $R_{1}$ and Rule $R_{2}$. We call such a set a power edge set of $G$ (see Fig. 1 for a guided example of RULE $R_{1}$ and RULE $R_{2}$ on a simple graph, leading to an optimal solution with two PMUs) and we let $p m u(G)$ denote the smallest size of any power edge set.

Power Edge Set (PES)

Input: a graph $G=(V, E)$ and some $k \in \mathbb{N}$

Question: Is $\operatorname{pmu}(G) \leq k$ ?

Previous work Toubaline et al. [15] propose a complexity result and an approximation threshold $1.12-\epsilon$ for $\epsilon>0$ based on an E-reduction from VERTEX Cover. They also propose a linear-time algorithm on trees by performing a polynomial reduction to PATH Cover. Moreover, Poirion et al. [14] develop an 
exact method, a linear program with binary variables, indexed on the necessary iterations using propagation RULE $R_{1}$ and RULE $R_{2}$, extended to a linear program in mixed variables, with the goal of being efficient in practice.

Our contribution An interesting open question stems from the assumption that power lines run in a plane or, at least in few planes or surfaces of low genus. In this work, we address this question, developing hardness results on (bipartite) planar graphs, covering both approximation and parameterized complexity. We show that PES is hard to approximate within a factor lower than $328 / 325$ for bipartite graphs (resp. $17 / 15-\epsilon)$, unless $\mathcal{P}=\mathcal{N} \mathcal{P}$, (resp. under $\mathcal{U G C})$. We also show that, assuming $\mathcal{E} \mathcal{T H}$, there is no $2^{o(\sqrt{n})}$-time algorithm, and no $2^{o(k)} n^{O(1)}$ time parameterized algorithm with respect to the standard parameter.

\section{Preliminaries}

In this section, we present some definitions and observations concerning parts of optimal solutions to PES on a graph $G$. We call a cycle $C$ ribbon if all but exactly one vertex $v$ of $C$ have degree two in $G$ and we call $v$ the knot of $C$.

Lemma 1. Let $G$ be a graph, let $C$ be a ribbon with knot $v$ and let $e$ be an edge of $C$. Then, there is an optimal power edge set $S$ for $G$ with $S \cap E(C)=\{e\}$.

Proof. Suppose that no PMU is placed on the edges of $C$. Then, even if $c(v)=$ 1 , none of the neighbors of $v$ in $C$ can become colored and, thus, $v$ cannot propagate on any of them. If one PMU is placed on $e$, we obtain $c(V(C))=\{1\}$ by consecutive propagation of vertices of degree two.

Definition 1 (Passive Relay). Let $G$ be a graph, let $C$ be a ribbon with knot $v$, and let $N_{G}(v) \backslash V(C)=\{x, y\}$. Then, $v$ is called passive relay between $x$ and $y$.

If $v$ is a passive relay between $x$ and $y$, then $c(x)=1$ implies $c(y)=1$ by Rule $R_{2}$ applied to $v$. A passive relay between $x$ and $y$ can be built by connecting $x$ and $y$ to a ribbon (see Figure 2). The interest of adding this relay lies in the fact that, by Lemma 1, any optimal power edge set intersects the ribbon, thus coloring it completely. Then, a coloration of $x$ necessarily implies a coloration of $y$ even if there were remaining uncolored vertices in $N_{G}(x)$ (and symmetrically from $y$ to $x$ ).

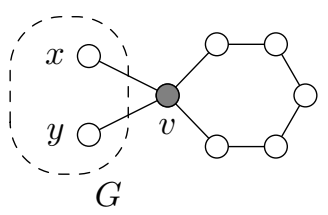

Fig. 2. A passive relay between $x$ and $y$, consisting in a ribbon with $\operatorname{knot} v$.

Throughout this work, we call a total order $<$ of vertices of $G$ valid for any $S \subseteq E(G)$ if, for each $v \in V(G)$, there is an edge incident with $v$ in $S$ or there is some $u \in N_{G}(v)$ with $N_{G}[u] \leq v$ (where $\leq$ denotes the extension of $<$ by all reflexive pairs). Note that valid orders correspond to propagation processes of $S$ in $G$. We also represent a total order $<$ by a sequence $\left(v_{1}, v_{2}, \ldots\right)$ such that $v_{i}$ occurs before $v_{j}$ in the sequence if and only if $v_{i}<v_{j}$. 


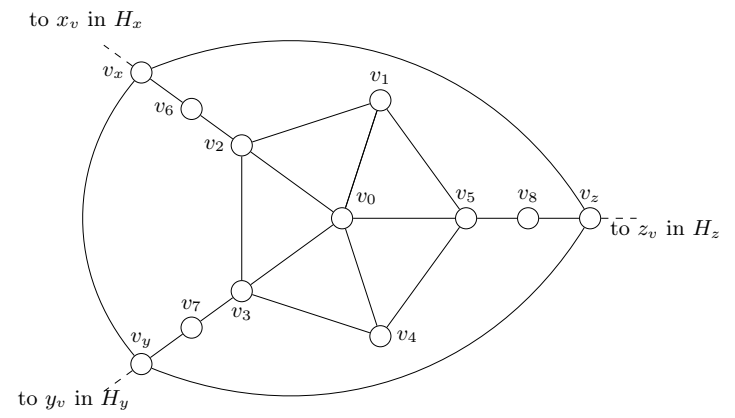

Fig. 3. Gadget $H_{v}$ for a vertex $v$ with neighbors $x, y$, and $z$.

\section{Computational results}

In this section, we present new complexity results for PES on restricted graphs. First, we show that PES remains $\mathcal{N} \mathcal{P}$-complete even if $G$ is a planar graph with bounded degree at most five (Theorem 1). Then, we extend this result to planar bipartite graphs with degree at most six (Theorem 2). To prove these results, we use a reduction from 3-REgular PLANAR VERTEX COVER (3-RPVC) defined as follows:

3-REgular Planar Vertex COVER (3-RPVC)

Input: a 3-regular planar graph $G=(V, E)$ and some $k \in \mathbb{N}$.

Question: Is there a size- $k$ set $S \subseteq V$ covering $E$, i.e. $\forall_{e \in E} e \cap S \neq \varnothing$ ?

3-RPVC is $\mathcal{N} \mathcal{P}$-complete [8] but admits a PTAS [1], and a $\frac{3}{2}$-approximation [3].

\subsection{Hardness on Planar Graphs}

First, we introduce the gadget graph $H_{v}$ presented in Figure 3:

Construction 1 Given a vertex $v$ of degree three with neighbors $x, y, z$, the gadget $H_{v}$ is composed of 1 ) an internal 5 -wheel (vertices $v_{0}-v_{5}$ ) with center $v_{0}$, 2) a set of three border-vertices, one for each neighbor of $v$, called $v_{x}, v_{y}$ and $v_{z}$ connected in a triangle 3) and three intermediate vertices $\left(v_{6}, v_{7}, v_{8}\right)$, connected respectively to $v_{x}$ and $v_{2}$, to $v_{y}$ and $v_{3}$, and to $v_{z}$ and $v_{5}$. The whole gadget contains 12 vertices and 19 edges.

From any 3-regular planar graph $G$, we construct a planar graph $G^{\prime}$ by 1. for each $v \in V(G)$, adding $H_{v}$, and 2. for each $\{u, v\} \in E$, adding a connecting edge $\left\{u_{v}, v_{u}\right\}$, thus linking the gadgets $H_{u}$ and $H_{v}$ (see Figure 6 (appendix)).

In the following, let $S^{\prime}$ be a solution to PES on $G^{\prime}$ and let $<$ be a valid order corresponding to $S^{\prime}$.

Lemma 2. $S^{\prime}$ contains an edge incident with $v_{0}, v_{1}$, or $v_{4}$ for all $v \in V(G)$. 
Proof. Towards a contradiction, assume that $S^{\prime}$ avoids all edges incident with $v_{0}, v_{1}$ and $v_{4}$ for some $v \in V(G)$. Then, since $v_{0}$ is neighbor of all neighbors (except $v_{0}$ itself) of $v_{1}$, we have $v_{0}<v_{1}$ and the same holds for $v_{4}$. However, all neighbors of $v_{0}$ have either $v_{1}$ or $v_{4}$ as a neighbor (or are $v_{1}$ or $v_{4}$ themselves), implying $v_{1}<v_{0}$ or $v_{4}<v_{0}$, contradicting $v_{0}<v_{1}, v_{4}$.

Lemma 3. For all $\{v, x\} \in E(G)$, we have $\left\{v_{x}, x_{v}\right\} \notin S^{\prime}$.

Proof. Towards a contradiction, assume that $\left\{v_{x}, x_{v}\right\} \in S^{\prime}$ for some $\{x, v\} \in$ $E(G)$. Then, we can swap $\left\{v_{x}, x_{v}\right\}$ and the edges in $S^{\prime} \cap E\left(H_{v}\right)$ for $\left\{v_{0}, v_{1}\right\}$ and $\left\{v_{4}, v_{5}\right\}$ in $S^{\prime}$, allowing us to start < with $\left(v_{0}, v_{1}, v_{4}, v_{5}, v_{2}, v_{3}, v_{6}, v_{7}, v_{8}, v_{x}\right.$, $\left.v_{y}, v_{z}, x_{v}\right)$ for $\{x, y, z\}=N_{G}(v)$. BY Lemma $2, S^{\prime}$ did not grow larger. Further, $v_{x}$ and $x_{v}$ precede all $w \notin V\left(H_{v}\right)$ in this modified ordering, implying that it is valid for the modified power edge set.

Lemma 4. Let $v \in V(G)$ with $\left|S^{\prime} \cap E\left(H_{v}\right)\right|=1$, let $x \in N_{G}(v)$ and let $w \in$ $\left\{v_{0}, v_{1}, \ldots, v_{8}\right\}$ such that $w$ is not incident with an edge of $S^{\prime}$. Then, $v_{x}<w$.

Proof. Abbreviate $B:=\left\{v_{i} \mid i \in N_{G}(v)\right\}$ and let $w$ be chosen minimal with respect to $<$. Since $w$ is not incident with an edge of $S^{\prime}$, there is some $u \in N_{G^{\prime}}(w)$ with $N_{G^{\prime}}[u] \leq w$. Assume towards a contradiction that $u \notin B$. By minimality of $w$, we then know that $u$ is incident with an edge of $S^{\prime}$ and by Lemma 2, $N_{G^{\prime}}[u]$ avoids $B$. However, since $\left|N_{G^{\prime}}[u]\right| \geq 4$ for all such $u$, this contradicts $\left|S^{\prime} \cap E\left(H_{v}\right)\right|=1$. Thus, $u \in B$, implying $v_{x} \in N_{G^{\prime}}[u]$ and $v_{x}<w$.

Lemma 5. Let $\{x, v\} \in E(G)$. Then $\left|S^{\prime} \cap E\left(H_{x}\right)\right|>1$ or $\left|S^{\prime} \cap E\left(H_{v}\right)\right|>1$.

Proof. Towards a contradiction, assume that $\left|S^{\prime} \cap E\left(H_{x}\right)\right|=\left|S^{\prime} \cap E\left(H_{v}\right)\right|=1$ (from Lemma 2, we know that $\left|S^{\prime} \cap E\left(H_{v}\right)\right| \geq 1$ ). By symmetry, suppose that $v_{x}<x_{v}$ and note that, by Lemma 2 and Lemma $3, v_{x}$ is not incident with an edge of $S^{\prime}$. Thus, there is some $u \in N_{G^{\prime}}\left(v_{x}\right)$ with $N_{G^{\prime}}[u] \leq v_{x}$. Since $v_{x}<x_{v}$, we have $u \in V\left(H_{v}\right)$. By Lemma 4 , we know that $u \in\left\{v_{i} \mid i \in N_{G}(v)\right\}$. However, $N_{G^{\prime}}[u]$ intersects $\left\{v_{0}, v_{1}, \ldots, v_{8}\right\}$, contradicting Lemma 4.

Theorem 1. Power Edge Set is $\mathcal{N} \mathcal{P}$-complete in planar graphs of degree at most five.

Proof. We show that $G$ has a size- $k$ vertex cover if and only if the result $G^{\prime}$ of applying Construction 1 has a power edge set of size $n+k$.

" $\Rightarrow$ ": let $S$ be a size- $k$ vertex cover of $G$. We build a power edge set $S^{\prime}$ for $G^{\prime}$ as follows: for each $v \in V(G)$, add the edge $\left\{v_{0}, v_{1}\right\}$ of $H_{v}$ to $S^{\prime}$ and for each $v \in S$, add the edge $\left\{v_{4}, v_{5}\right\}$ of $H_{v}$ to $S^{\prime}$. Note that $\left|S^{\prime}\right|=n+k$. We construct a valid ordering $<$ of $G^{\prime}$ for $S^{\prime}$. To this end, for each $v \in V(G)$ with $(x, y, z)$ being an arbitrary sequence of $N_{G}(v)$, let

$$
<_{v}:=\left\{\begin{array}{l}
\left(v_{0}, v_{1}, v_{4}, v_{5}, v_{2}, v_{3}, v_{6}, v_{7}, v_{8}, v_{x}, v_{y}, v_{z}\right) \text { if } v \in S \\
\left(v_{0}, v_{1}, v_{x}, v_{y}, v_{z}, v_{6}, v_{7}, v_{8}, v_{2}, v_{3}, v_{5}, v_{4}\right) \text { if } v \notin S .
\end{array}\right.
$$




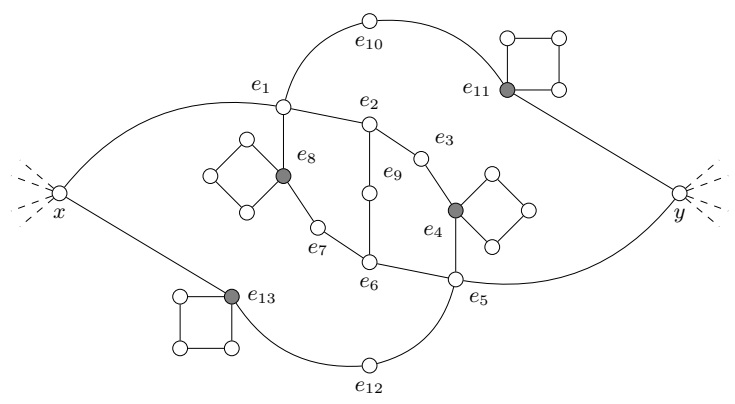

Fig. 4. Gadget graph $I(e)$ with $e=\{x, y\}$.

Let $<^{*}$ be an arbitrary ordering of $V(G)$ such that $u<^{*} v$ for all $u \in S$ and $v \notin S$ and let $<$ be the result of replacing each $v$ by the sequence $<_{v}$ in this ordering. Towards a contradiction, assume that $<$ is not valid for $S^{\prime}$ and let $w$ be the first vertex of $<$ such that the subsequence of $<$ ending with $w$ is invalid for $S^{\prime}$. Let $v \in V(G)$ such that $w$ is a vertex of $H_{v}$. By construction of $<_{v}$, this is only possible if $v \notin S$ and $w=v_{x}$ for some $x \in N_{G}(v)$. However, since $S$ is a vertex cover, $x \in S$, implying $x<^{*} v$ and, thus, $V\left(H_{x}\right)<w$. But then, $N_{G^{\prime}}\left[x_{v}\right] \leq v_{x}$ contradicting that the subsequence of $S^{\prime}$ ending with $w$ is invalid.

" $\Leftarrow$ ": Let $S^{\prime}$ be a size- $(n+k)$ power edge set of $G^{\prime}$ and let $<$ be a valid total order of $V\left(G^{\prime}\right)$ for $S^{\prime}$. Lemma 5 directly implies that the set $\left\{v|| S^{\prime} \cap E\left(H_{v}\right) \mid>1\right\}$ is a vertex cover of $G$ and, by Claims 2,4 and 5 , its size is at most $\left|S^{\prime}\right|-n=k$.

\subsection{Hardness on Bipartite Planar Graphs}

In the proof of Theorem 1, the graph $G^{\prime}$ obtained by Construction 1 is not bipartite. In the following, we modify this construction to yield planar bipartite graphs while preserving large parts of the previous proof. To this end, we replace edges of odd-length cycles with a gadget (See Figure 4) and show that this replacement does not alter the initial coloring propagation scheme in the graph.

Construction 2 Given a graph $G$ and an edge $e \in E(G)$, let $r(G, e)$ denote the graph $(V(G) \cup V(I(e)), E(G) \cup E(I(e)) \backslash e)$ resulting from replacing e by the gadget graph $I(e)$ in $G$ (see Figure 4).

Note that $I(e)$ is bipartite and planar, and that the distance between $x$ and $y$ is even. By Lemma 1, we know that each of the four 4-cycles connected to $e_{4}$, $e_{8}, e_{11}$, and $e_{13}$, respectively contains a PMU. Moreover, vertex $e_{4}$ (respectively $\left.e_{8}, e_{11}, e_{13}\right)$ is a passive relay between $e_{3}$ and $e_{5}$ (respectively between $e_{1}$ and $e_{7}$, between $e_{10}$ and $y$, between $e_{12}$ and $x$ ). Recall that one can consider passive relays and their connected cycles as always colored.

Lemma 6. Let $G$ be a graph, let $e=\{x, y\} \in E(G)$, and let $G^{\prime}=r(G, e)$. Then, $\operatorname{pmu}(G) \leq k$ if and only if pmu $\left(G^{\prime}\right) \leq k+4$. 
Proof. " $\Rightarrow$ ": Let $F_{e}$ be a set containing one edge of each ribbon of $I(e)$, let $S$ be a size- $k$ power edge set for $G$, and let $S^{\prime}:=(S \backslash\{x, y\}) \cup F_{e}$. We suppose that $\{x, y\} \notin S$ as otherwise, $S^{\prime} \cup\left\{x, e_{1}\right\}$ is a power edge set for $G$ and its size is $k+4$. Let $<$ be a valid order of $G$ for $S$ and let $\left(v_{1}, v_{2}, \ldots\right)$ be the sequence of $V(G)$ corresponding to $<$. From <, we build a valid ordering $<^{\prime}$ of $G^{\prime}$ for $S^{\prime}$, thus proving that $S^{\prime}$ is a power edge set for $G^{\prime}$. Without loss of generality, let $x<y$ and note that $\left(v_{1}, v_{2}, \ldots, x\right)$ is valid for $S^{\prime}$. Let $z$ be minimal with respect to $<$ such that $N_{G}[x] \leq z$ and let $<^{\prime}$ be the result of (1) prepending the vertices of the ribbons of $I(e)$ to $<,(2)$ replacing $x$ by $\left(x, e_{12}, e_{5}, e_{3}, e_{2}\right),(3)$ replacing $z$ by $\left(e_{1}, e_{7}, e_{6}, e_{9}, e_{10}, z\right)$ if $z=y$, and (4) replacing $y$ by $\left(y, e_{10}, e_{1}, e_{7}, e_{6}, e_{9}\right)$ if $z \neq y$. Let $\left(v_{1}^{\prime}, v_{2}^{\prime}, \ldots\right)$ be the corresponding vertex sequence. Towards a contradiction, assume that there is some $w$ such that $\left(v_{1}^{\prime}, v_{2}^{\prime}, \ldots, w\right)$ is not valid for $S^{\prime}$ and let $w$ be minimal with respect to $<^{\prime}$. As $w$ is not incident with an edge of $S^{\prime}$, it is also not incident with an edge of $S$. Further, one can verify that (1)-(4) imply $w \neq e_{j}$ for all $j$ and, thus, $w \in V(G)$. Since $<$ is valid for $S$, there is some $u \in N_{G}(w)$ with $N_{G}[u] \leq w$. First, suppose that $u=x$ and note that $x, y \leq w=z$ in this case. If $y=w=z$, then $N_{G}[x] \leq y$ and $N_{G^{\prime}}\left[e_{11}\right] \leq^{\prime} y$ by (3). Otherwise, $y<w$ and, by (4), $e_{1}, e_{13}<^{\prime} w$, implying $N_{G^{\prime}}[u] \leq^{\prime} w$. Second, suppose that $u=y$. By (1) and (2), however, $e_{5}, e_{11}<^{\prime} y<^{\prime} w$, implying $N_{G^{\prime}}[u] \leq^{\prime} w$. Thus, $u \notin V(I(e))$, implying $N_{G}[u]=N_{G^{\prime}}[u]$ and $N_{G^{\prime}}[u] \leq^{\prime} w$ as $<^{\prime}$ is an extension of $<$.

" $\Leftarrow$ ": Let $S^{\prime}$ be a size- $(k+4)$ power edge set for $G^{\prime}$ and let $S_{e}^{\prime}:=S^{\prime} \cap E(I(e))$. If $\left|S_{e}^{\prime}\right| \geq 5$, then $(S \backslash E(I(e))) \cup\{\{x, y\}\}$ is clearly a power edge set for $G$ and its size is at most $k$. Otherwise, $\left|S_{e}^{\prime}\right| \leq 4$ and, by Lemma $1, S_{e}^{\prime}$ consists of four edges; one in each ribbon of $I(e)$. Let $S:=S^{\prime} \backslash S_{e}^{\prime}$, let $<^{\prime}$ be a valid order of $G^{\prime}$ for $S^{\prime}$, and let $<$ be the restriction of $<^{\prime}$ to $V(G)$. Let $\left(v_{1}^{\prime}, v_{2}^{\prime}, \ldots\right)$ and $\left(v_{1}, v_{2}, \ldots\right)$ be the sequences of $V\left(G^{\prime}\right)$ and $V(G)$ implied by $<^{\prime}$ and $<$, respectively. Without loss of generality, let $x<^{\prime} y$, implying $x<y$. By construction of $I(e)$, we observe that $S_{e}$ does not propagate beyond the ribbons of $I(e)$, implying that

$$
\forall_{i \in\{1,2,3,5,6,7,9,10,12\}} x<^{\prime} e_{i} \text { and } \forall_{i \in\{1,6,7,9,10\}}\left(N_{G^{\prime}}[x] \leq^{\prime} e_{i}\right) \vee\left(y<^{\prime} e_{i}\right) \text {. }
$$

We show that $<$ is valid for $S$. Towards a contradiction, assume that there is some $w \in V(G)$ such that $\left(v_{1}, v_{2}, \ldots, w\right)$ is not valid for $S$ and let $w$ be minimal with respect to $<$. Since $w \in V(G)$ and it is not incident with any edges of $S$, it is also not incident with any edges of $S^{\prime}$, implying that there is some $u \in V\left(G^{\prime}\right)$ with $N_{G^{\prime}}[u] \leq^{\prime} w$. First, suppose that $u \in V\left(G^{\prime}\right) \backslash V(G)$ and since, by (1), $w \neq x$ , we have $w=y$ and $u \in\left\{e_{5}, e_{11}\right\}$. Thus, $N_{G^{\prime}}\left[e_{5}\right] \leq^{\prime} y$, implying $e_{6}<^{\prime} y$ or $N_{G^{\prime}}\left[e_{11}\right] \leq^{\prime} y$, implying $e_{10}<^{\prime} y$. In either case, (1) implies $N_{G^{\prime}}[x] \leq^{\prime} y$ and, thus, $N_{G}[x] \leq y$. Second, suppose that $u \in V(G)$. Since $N_{G}[u]=N_{G^{\prime}}[u]$ for all $u \in V(G) \backslash\{x, y\}$, we have $u \in\{x, y\}$ as otherwise, $N_{G}[u] \leq w$. If $u=y$, then $N_{G}[u] \leq w$ since $N_{G}[y]=\left(N_{G^{\prime}}[y] \cap V(G)\right) \cup\{x\}$. If $u=x$ then, since $e_{1} \in N_{G^{\prime}}[u]$, we have $e_{1}<^{\prime} w$. But since $w \in N_{G^{\prime}}[x]$, we have $N_{G^{\prime}}[x] \not \mathbb{L}^{\prime} e_{1}$ and (1) implies $y<^{\prime} e_{1}$. As $N_{G}[x]=\left(N_{G^{\prime}}[x] \cap V(G)\right) \cup\{y\}$, we conclude $N_{G}[x] \leq w$.

In order to show hardness on bipartite graphs, we color the vertices of the output graph $G^{\prime}$ of Construction 1 arbitrarily with two colors and replace all 
monochromatic edges $e$ with $I(e)$. We can strengthen the result using the following coloring strategy. For each boundary vertex $v_{i}$ of each $H_{v}$, color $v_{i}$ such that $N_{G^{\prime}}\left[v_{i}\right] \backslash\left\{v_{6}, v_{7}, v_{8}\right\}$ is not monochromatic and let $c$ be the color occurring the least among $\left\{v_{x}, v_{y}, v_{z}\right\}$. Then, color $v_{0}, v_{6}, v_{7}$, and $v_{8}$ with $c$ and color $v_{1}-v_{5}$ with the other color.

Lemma 7. In $H_{v}$, each $v_{i}$ with $i \in\{x, y, z\}$ is incident with at most two monochromatic edges.

Proof. Let the color of $v_{x}$ be blue and assume towards a contradiction that $v_{x}$ is incident with at least three monochromatic edges. As $N_{G^{\prime}}\left[v_{x}\right] \backslash\left\{v_{6}\right\}$ is not monochromatic, $v_{6}$ is blue. But then, blue appears least among $v_{x}, v_{y}, v_{z}$, implying that $v_{y}$ and $v_{z}$ are not blue. Thus, $v_{x}$ is incident with at most two monochromatic edges.

Considering Lemma 7, we observe that the graph resulting from replacing monochromatic edges of $G^{\prime}$ has maximum degree six.

Theorem 2. Power Edge Set is $\mathcal{N} \mathcal{P}$-complete in planar bipartite graphs of degree six.

\section{Some Lower Bounds}

\subsection{Non-Approximability}

In this section, we prove new approximation lower bounds for PES, improving the current best known bounds presented by Toubaline et al. [15]. First recall the definition of $L$-reduction between two difficult problems $\Pi$ and $\Pi^{\prime}$, described by Papadimitriou and Yannakakis [13]. This reduction consists of polynomialtime computable functions $f$ and $g$ such that, for each instance $x$ of $\Pi, f(x)$ is an instance of $\Pi^{\prime}$ and for each feasible solution $y^{\prime}$ for $f(x), g\left(y^{\prime}\right)$ is a feasible solution for $x$. Moreover there are constants $\alpha_{1}, \alpha_{2}>0$ such that:

1. $O P T_{\Pi^{\prime}}(f(x)) \leq \alpha_{1} O P T_{\Pi}(x)$ and

2. $\left|\operatorname{val}_{\Pi}\left(g\left(y^{\prime}\right)\right)-O P T_{\Pi}(x)\right| \leq \alpha_{2}\left|v a_{\Pi^{\prime}}\left(y^{\prime}\right)-O P T_{\Pi^{\prime}}(f(x))\right|$.

We use an $L$-reduction from VERTEX COVER in hypergraphs in which all edges have cardinality exactly 3 .

\section{3-UNIFORM VC (3UVC)}

Input: a 3-uniform hypergraph $G=(V, E)$ and some $k \geq 2$.

Question: Is there a size- $k$ vertex set $V^{\prime} \subseteq V$ covering $E$ ?

3-UNIFORM VC is hard to approximate within a factor less than $2-\epsilon$ for all $\epsilon>0$, unless $\mathcal{P}=\mathcal{N} \mathcal{P}$, even if each vertex appears in at most three edges [6]

Theorem 3. Under $\mathcal{U G C}$, Power Edge Set is hard to approximate within a factor of $\frac{17}{15}-\epsilon$, even on graphs of maximum degree five. 


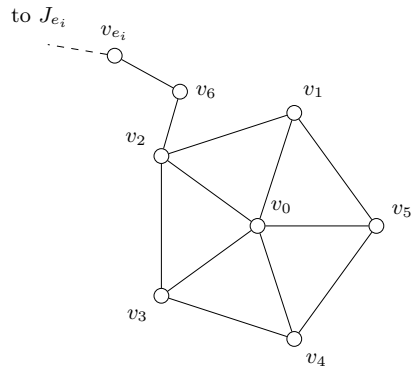

(a) $H_{v}^{1}$-gadget $\forall v \in V$ included into one edge $e_{i}$ ).

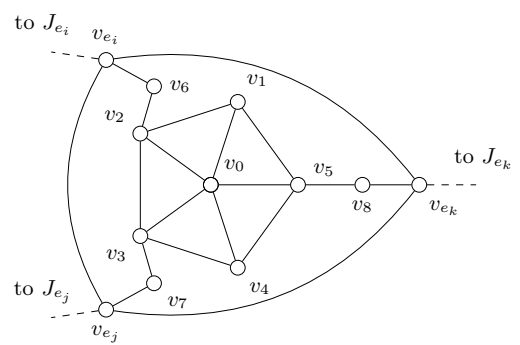

(c) $H_{v}^{3}$-gadget $\forall v \in V$ included into three edges $\left.e_{i}, e_{j}, e_{k}\right)$.

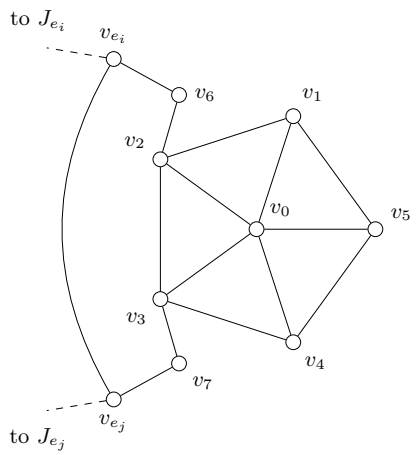

(b) $H_{v}^{2}$-gadget $\forall v \in V$ included into two edges $e_{i}$ and $e_{j}$ ).

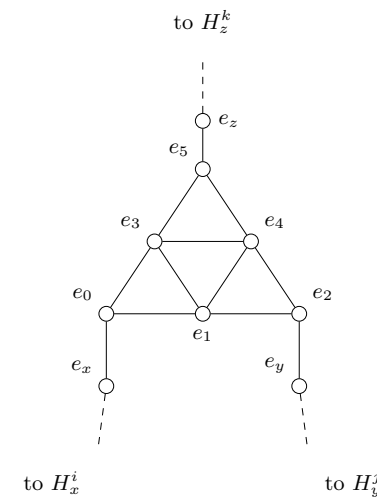

(d) $J_{e}$-gadget: $\forall e \in E$ with $e=\{x, y, z\}$

Fig. 5. Polynomial-time reduction for hypergraph $G$.

Proof. Given an instance $I=(G, k)$ of 3-UnIform VC such that each vertex of $G$ appears in at most three edges, we construct an instance $I^{\prime}=\left(G^{\prime}, k+n+m\right)$ of PES in the following way: For each $v \in V$ included in exactly $\gamma \leq 3$ edges, we add a gadget $H_{v}^{\gamma}$ given in Fig. 5a-5c. Vertices $v_{e_{i}}, v_{e_{j}}, v_{e_{k}}$ are border-vertices for $H_{v}^{1}, H_{v}^{2}, H_{v}^{3}$. For each hyperedge $e=\{x, y, z\}$, we add a gadget $J_{e}$, given by Fig. $5 \mathrm{~d}$ with border vertices $e_{x}, e_{y}, e_{z}$ and we add the edges $\left\{x_{e}, e_{x}\right\},\left\{y_{e}, e_{y}\right\}$, and $\left\{z_{e}, e_{z}\right\}$.

Vertex-gadgets $H_{v}^{\gamma}$ are designed such that if we have $c\left(v_{e_{j}}\right)=1$ for all $e_{j} \in E$ containing $v$, then placing a single PMU inside $H_{v}^{\gamma}$ is sufficient to color the whole vertex-gadget. If $c\left(e_{v}\right)=0$ for some $e$ containing $v$, two PMUs are necessary in $H_{v}^{\gamma}$ to color the whole gadget, but this also colors $e_{v}^{\prime}$ for all $e^{\prime}$ containing $v$. Edgegadgets $J_{e}$ are designed such that if at least one border vertex $e_{x}$ is colored, then only one PMU is required in $J_{e}$ to color the whole edge-gadget, but this also 
colors $v_{e}$ for all $v \in e$. Note that if there are two PMUs on any edge-gadget $J_{e}$ in an optimal solution, then one can simply switch one PMU from $J_{e}$ to any adjacent $H_{v}^{\gamma}$ and get a solution of same cost with only one PMU per edge-gadget.

Observe that $G$ admits a size- $k$ vertex cover if and only if $G^{\prime}$ can be monitored with $k+n+m$ PMUs: the vertex-gadgets $H_{v}^{\gamma}$ with two PMUs propagate on the border-vertices on all edge-gadgets. If we add one PMU per edge-gadget, any colored border vertex of $H_{v}^{\gamma}$ propagates its color to all other border vertices. To show that the vertex-gadgets $H_{v}^{\gamma}$ with two PMUs induce a vertex cover of $I$, suppose that there is a hyperedge $e=\{u, v, w\} \in E$ that is not covered. Then, their respective vertex gadgets contain a single PMU. Then, however, these vertex gadgets cannot be colored by Rule $R_{2}$, contradicting $I^{\prime}$ being monitored.

To show that the above constitutes an $L$-reduction, let $f$ be a function transforming any instance $I$ of 3-UNIFORM VC into an instance $I^{\prime}$ of pmuas above, let $S^{\prime}$ be any feasible solution for $I^{\prime}$, and let $g$ be the function that transforms $S^{\prime}$ into a solution $S^{\prime \prime}$ that contains exactly one edge of each $J_{e}$ and at least one edge of each $H_{v}^{\gamma}$, and then outputs the set of vertices $v$ for which $S^{\prime \prime}$ assigns at least two PMUs to $H_{v}^{\gamma}$. First, the above argument shows that $g\left(S^{\prime}\right)$ is a feasible solution for 3-UNIFORM VC. Second, by construction,

$$
O P T\left(I^{\prime}\right)=O P T(I)+n+m
$$

and, since each vertex of $I$ appears in at most 3 edges of $I$, at least one in seven vertices has to be in a vertex cover of $G$, implying $n / 7 \leq O P T(I)$. Since each vertex is incident with at most three hyperedges and each hyperedge contains exactly three vertices, Hall's theorem implies $m \leq n$. We then obtain $O P T\left(I^{\prime}\right) \leq$ $15 \cdot O P T(I)$. Third, by construction of $g$, we have

$$
\operatorname{val}\left(g\left(S^{\prime}\right)\right) \leq \operatorname{val}\left(S^{\prime}\right)-m-n \stackrel{(2)}{\leq} \operatorname{val}\left(S^{\prime}\right)-O P T\left(I^{\prime}\right)+O P T(I)
$$

Thus, we constructed an $L$-reduction with $\alpha_{1}=15, \alpha_{2}=1$. Assuming $\mathcal{U G C}$, 3 -UNIFORM $\mathrm{VC}$ is hard to approximate to a factor of $(3-\epsilon)[2]$ and, thus

$$
\begin{aligned}
\operatorname{val}\left(S^{\prime}\right) & \stackrel{(3)}{\geq} \operatorname{val}\left(g\left(S^{\prime}\right)\right)+\operatorname{OPT}\left(I^{\prime}\right)-O P T(I) \\
& \geq 3 \cdot O P T(I)+O P T\left(I^{\prime}\right)-O P T(I) \\
& \geq 2 / 15 \cdot O P T\left(I^{\prime}\right)+O P T\left(I^{\prime}\right) \\
& \geq 17 / 15 \cdot O P T\left(I^{\prime}\right)
\end{aligned}
$$

Theorem 4. Power EDGE SET on bipartite graphs of maximum degree six cannot be approximated to within a factor better than 328/325 $>1.0092$ unless $\mathcal{P}$ $=\mathcal{N} \mathcal{P}$.

Proof. To show that the reduction from 3-RPVC presented in Construction 2 is an $L$-reduction, let $I$ be an instance of 3 -RPVC, let $f$ be the described reduction and let $g$ be the function that, given any feasible solution $S^{\prime}$ for $I^{\prime}:=f(I)$, 
transforms $S^{\prime}$ into a feasible solution $S^{\prime \prime}$ according to Lemma 2-5 and returns the set of vertices $v$ such that $S^{\prime \prime}$ contains at least two edges more than four times the number of gadgets $I(e)$ in $H_{v}$. Let $m^{\prime}$ be the total number of edges $e$ that are replaced by $I(e)$ by $f$. Using similar arguments, as in the proof of Theorem 3 we have $O P T\left(I^{\prime}\right)=O P T(I)+n+4 m^{\prime}$ and, since the graph $G$ of $I$ is 3 -regular, $n / 2 \leq O P T(I)$ (no independent set of $G$ can be larger than $n / 2$ ). Additionally to the coloring scheme suggested to prove Lemma 7 , we repeatedly find a $H_{v}$ with at least two incident inter-gadget edges that are monochrome and swap the coloring of $H_{v}$. Then, $m^{\prime} \leq 4 n+m \leq 4 n+m / 3=4 n+n / 2$, we further have $O P T\left(I^{\prime}\right) \leq 39 \cdot O P T(I)$. Then, $\operatorname{val}\left(S^{\prime}\right) \geq \operatorname{val}\left(g\left(S^{\prime}\right)\right)+O P T\left(I^{\prime}\right)-O P T(I)$. Since VERTEX COVER is hard to approximate to within a factor of 1.36 , even in 3-regular graphs $[5,7]$ (unless $\mathcal{P}=\mathcal{N} \mathcal{P})$, we conclude $\operatorname{val}\left(S^{\prime}\right) \geq 328 / 325 O P T\left(I^{\prime}\right)$.

\subsection{Lower Bounds for Exact and FPT Algorithms}

We propose some negative results for POWER EDGE SET about the existence of subexponential-time algorithms under $\mathcal{E} \mathcal{T} \mathcal{H}[9,10]$, and $\mathcal{F} \mathcal{P} \mathcal{T}$ Algorithms. Since the polynomial-time transformation given in the proof of Theorem 1 is linear in the number of vertices, and since 3-REgular PLANAR VERTEX Cover does not admit a $2^{o(\sqrt{n})} n^{O(1)}$-time algorithm [7, 11], there is also no $2^{o(\sqrt{n})} n^{O(1)}$-time algorithm for Power EDGE SET. Moreover, since the solution size $k$ is at most $n$, a $2^{o(k)} n^{O(1)}$-time algorithm contradicts the non-existence (assuming $\mathcal{E} \mathcal{T H}$ ) of $2^{o(n)} n^{O(1)}$-time algorithms for VERTEX COVER on planar graphs [11].

Corollary 1. Assuming $\mathcal{E} \mathcal{T H}$, there is no $2^{o(\sqrt{n})} n^{O(1)}$-time algorithm for PowER EDGE SET in planar graphs, and there is no $2^{o(k)} n^{O(1)}$-time algorithm for POWER EDGE SET where $k$ is the solution size.

\section{Conclusion}

In this article, we presented several new hardness results and some lowers bounds for the problem of selecting a smallest number of phasor measurement units to monitor a given (planar) network. As perspectives, it would be interesting to explore the problem on particular classes of graphs to understand to what extend the regularity of the graph, or special patterns and minors, may influence the complexity of the problem. Further, having excluded $2^{o(k)} n^{O(1)}$-time algorithms, it is also interesting to seek "the next best thing", that is, single exponentialtime algorithms with respect to $k$ as well as considering structural parameters that are independent of planarity, such as the treewidth. Finally, as the problem is hard to approximate in polynomial time, it is interesting to allow moderately exponential time, in an $\mathcal{F} \mathcal{P} \mathcal{T}$-approximation setting (see $[4,12]$ ). 


\section{Bibliography}

[1] B. S. Baker. Approximation Algorithms for NP-Complete Problems on Planar Graphs. Journal of ACM, 41(1):153-180, 1994.

[2] N. Bansal and S. Khot. Inapproximability of hypergraph vertex cover and applications to scheduling problems. In Proc. 37th ICALP, pages 250-261, 2010.

[3] R. Bar-Yehuda and S. Even. On Approximating a Vertex Cover for Planar Graphs. In Proc. 14th STOC, pages 303-309, 1982.

[4] C Bazgan. Approximation schemes and parameterized complexity. $\mathrm{PhD}$ thesis, PhD thesis, INRIA, Orsay, France, 1995.

[5] I. Dinur and S. Safra. On the hardness of approximating vertex cover. Annals of Mathematics, 162(1):439-485, 2005. ISSN 0003-486X. doi: 10. 4007/annals.2005.162.439.

[6] I. Dinur, V. Guruswami, S. Khot, and O. Regev. A new multilayered PCP and the hardness of hypergraph vertex cover. SIAM Journal on Computing, 34(5):1129-1146, 2005.

[7] U. Feige. Vertex cover is hardest to approximate on regular graphs. Technical Report MCS03-15, the Weizmann Institute, 2003.

[8] M. R. Garey and D. S. Johnson. Computers and Intractability: A Guide to the Theory of NP-Completeness. W. H. Freeman \& Co., New York, NY, USA, 1979. ISBN 0716710447.

[9] R. Impagliazzo and R. Paturi. On the Complexity of $k$-SAT. Journal of Computer and System Sciences, 62(2):367-375, 2001.

[10] R. Impagliazzo, R. Paturi, and F. Zane. Which Problems Have Strongly Exponential Complexity? Journal of Computer and System Sciences, 63(4): 512-530, 2001.

[11] D. Lokshtanov, D. Marx, and S. Saurabh. Lower bounds based on the Exponential Time Hypothesis. Bulletin of the EATCS, 105:41-72, 2011.

[12] Dániel Marx. Parameterized complexity and approximation algorithms. The Computer Journal, 51(1):60-78, 2008.

[13] C. H. Papadimitriou and M. Yannakakis. Optimization, approximation, and complexity classes. Journal of Computer and System Sciences, 43(3): 425-440, 1991.

[14] P.-L. Poirion, S. Toubaline, C. D'Ambrosio, and L. Liberti. The power edge set problem. Networks, 68(2):104-120, 2016.

[15] S. Toubaline, C. D'Ambrosio, L. Liberti, P.-L. Poirion, B. Schieber, and H. Shachnai. Complexité du problème power edge set. In Roadef 2016, 2016.

[16] W. Yuill, A. Edwards, S. Chowdhury, and S. P. Chowdhury. Optimal pmu placement: A comprehensive literature review. In 2011 IEEE Power and Energy Society General Meeting, pages 1-8, July 2011. doi: 10.1109/PES. 2011.6039376. 


\section{Appendix}

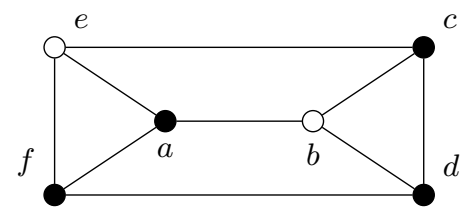

(a) A 3-regular planar graph $G$ and an optimal solution $\mathrm{S}=\{\mathrm{a}, \mathrm{c}, \mathrm{d}, \mathrm{f}\}$ to Vertex-Cover

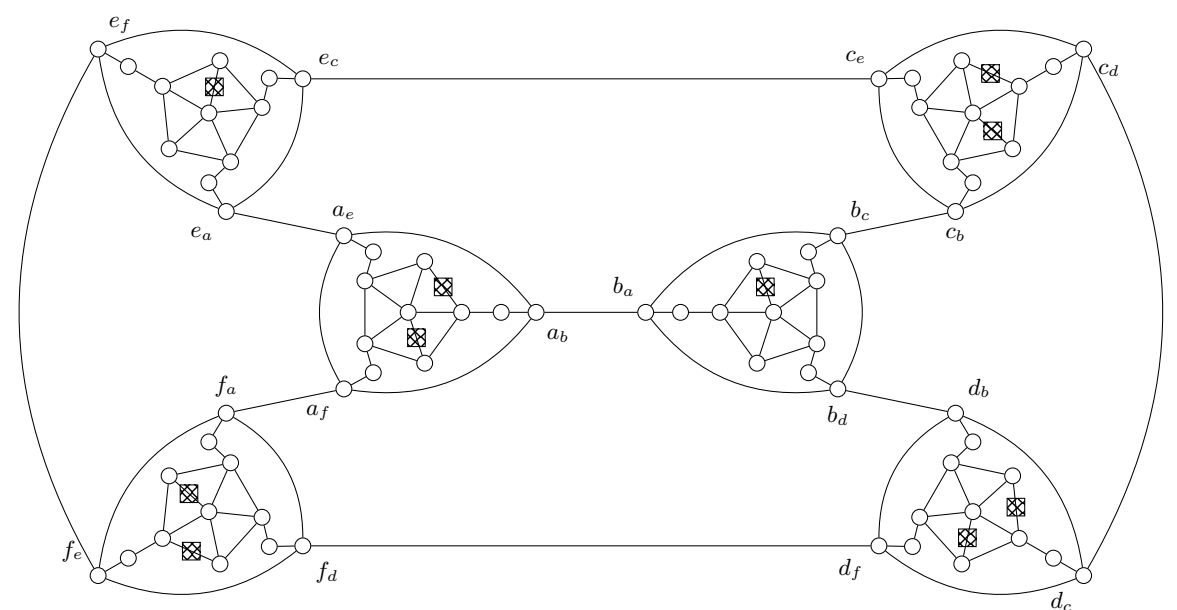

(b) The graph $G^{\prime}$ obtained from $G$ and the solution $S^{\prime}$ obtained from $S$. Here PMU are placed on the edges with boxes

Fig. 6. Example of a graph constructed from an instance $I$ of 3-RPVC (Proof of Theorem 1) 


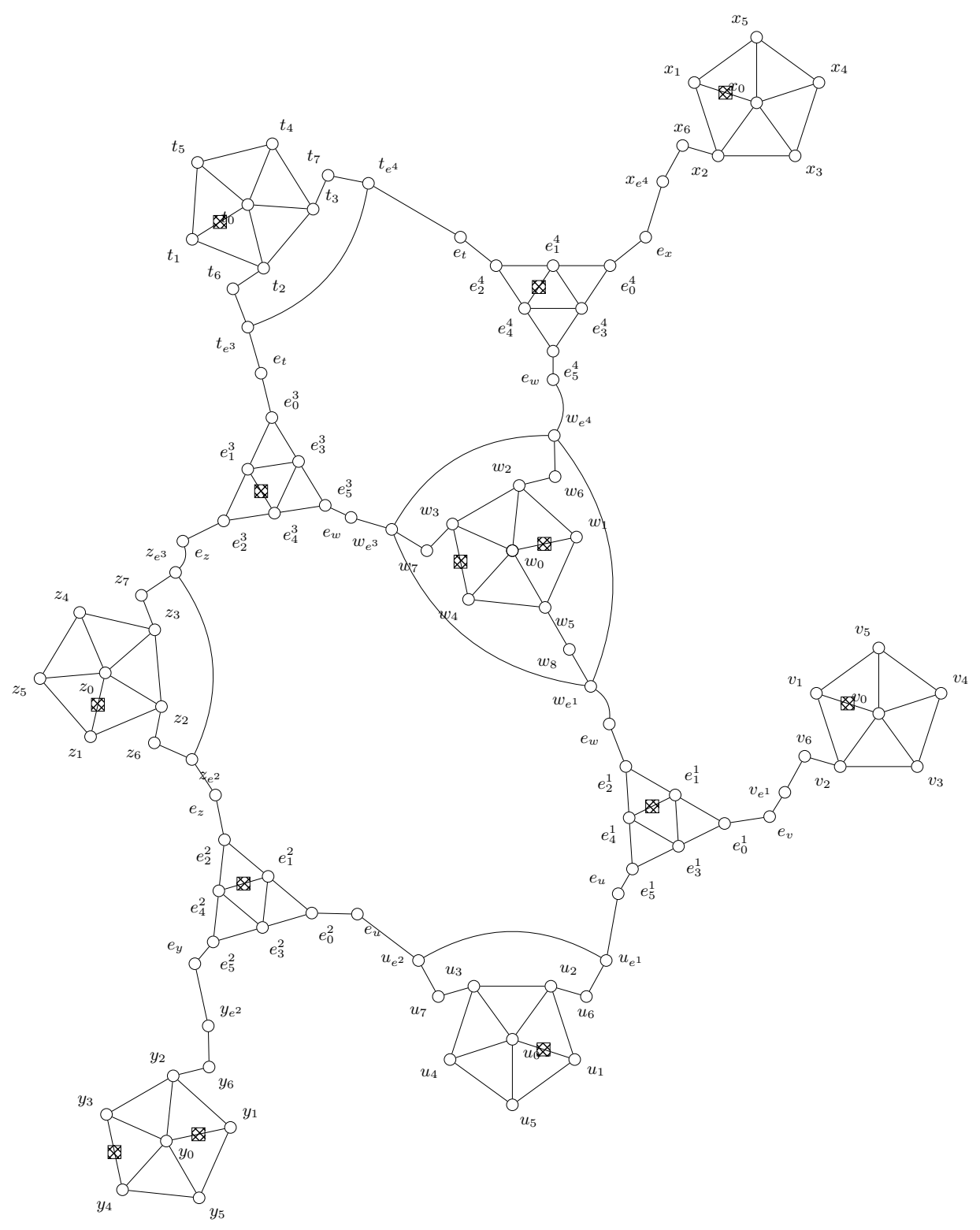

Fig. 7. Graph constructed from an instance $I$ of ERVC with $r=3$ (Proof of Theorem 3). The 3-uniform hypergraph from $I$ contains 8 vertices $t, u, v, w, x, y, z$ and the four edges $e^{1}=\{u, v, w\}, e^{2}=\{u, y, z\}, e^{3}=\{t, w, z\}, e^{4}=\{t, w, x\}$. An optimal solution for PES is to place PMUs on edges with a box. VertexGadgets $w$ and $y$ are the only one with two PMU. Thus $\{w, y\}$ is a vertex cover in the hypergraph. 